DOI: $10.17516 / 1997-1397-2020-13-2-187-196$

УДК $517.55+517.962 .26$

\title{
The Cauchy Problem for Multidimensional Difference Equations in Lattice Cones
}

\author{
Alexander P. Lyapin* \\ Siberian Federal University \\ Krasnoyarsk, Russian Federation \\ Lesosibirsk Pedagogical Institute - branch of SFU \\ Lesosibirsk, Russian Federation \\ Sreelatha Chandragiri ${ }^{\dagger}$ \\ Siberian Federal University \\ Krasnoyarsk, Russian Federation
}

Received 21.12.2019, received in revised form 26.01.2020, accepted 03.02.2020

\begin{abstract}
We consider a variant of the Cauchy problem for a multidimensional difference equation with constant coefficients, which connected with a lattice path problem in enumerative combinatorial analysis. We obtained a formula in which generating function of the solution to the Cauchy problem is expressed in terms of generating functions of the Cauchy data and a formula expressing solution to the Cauchy problem through its fundamental solution and Cauchy data.
\end{abstract}

Keywords: difference equation, fundamental solution, generating function, Dyck paths.

Citation: A.P.Lyapin, S.Chandragiri, The Cauchy Problem for Multidimensional Difference Equations in Lattice Cones, J. Sib. Fed. Univ. Math. Phys., 2020, 13(2), 187-196.

DOI: 10.17516/1997-1397-2020-13-2-187-196.

\section{Definitions and main results}

On complex valued functions $f: \mathbb{Z}^{n} \rightarrow \mathbb{C}$ we define the shift operator $\delta_{j}$ as follows:

$$
\delta_{j}: f\left(x_{1}, \ldots, x_{j}, \ldots, x_{n}\right) \mapsto f\left(x_{1}, \ldots, x_{j}+1, \ldots, x_{n}\right)
$$

and the polynomial difference operator

$$
P(\delta)=\sum_{\omega \in \Omega} c_{\omega} \delta^{\omega}
$$

where $\Omega \subset \mathbb{Z}^{n}$ is a finite set of points of an $n$-dimensional lattice, $\delta^{\omega}=\delta_{1}^{\omega_{1}} \cdot \ldots \cdot \delta_{n}^{\omega_{n}}$ and $c_{\omega} \in \mathbb{C}$ are the coefficients of the difference operator.

We consider the difference equation

$$
P(\delta) f(x)=g(x), \quad x \in X,
$$

where $f(x)$ is an unknown function, and $g(x)$ is a function defined on some set $X \subset \mathbb{Z}^{n}$. Also choose a set $X_{0} \subset \mathbb{Z}^{n}$, the points of which will be called initial (boundary) points.

\footnotetext{
*aplyapin@sfu-kras.ruｈttps://orcid.org/0000-0002-0149-7587

†srilathasami66@gmail.com

(c) Siberian Federal University. All rights reserved
} 
In the general situation we have to solve the Cauchy problem: find a function $f(x)$, satisfying equation (1) and coinciding with a given function $\varphi(x)$ of initial data on the set $X_{0}$ :

$$
f(x)=\varphi(x), \quad x \in X_{0} .
$$

The function $g(x)$ in the right-hand side of $(1)$ and the initial data function $\varphi(x)$ in $(2)$ is called the Cauchy data of problem (1)-(2).

Existence and uniqueness of problem (1)-(2) (solvability of the Cauchy problem) depends on all the objects involved in its formulation: the difference operator $P(\delta)$, the set $X$ on which the right part of the equation is given, and the set $X_{0}$ on which the initial data $\varphi(x)$ is defined.

In the one-dimensional case two variants of the Cauchy problems are usually considered:

(i) $X=\{x \in \mathbb{Z}: x \geqslant 0\}$ is the set of non-negative integers, $P(\delta)=\sum_{\omega=0}^{m} c_{\omega} \delta^{\omega}, X_{0}=$ $=\{0,1, \ldots, m-1\}, c_{m} \neq 0$,

(ii) $X=\{x \in \mathbb{Z}: x \geqslant m\}, P(\delta)=\sum_{\omega=0}^{m} c_{\omega} \delta^{-\omega}, X_{0}=\{0,1, \ldots, m-1\}, c_{0} \neq 0$.

For example, option (i) is used to describe the solution to equation (1) in the theory of discrete dynamic systems (see [6]). Option (ii) is most useful in problems of enumerative combinatorial analysis (see [17]).

In the case of constant coefficients, the $z$-transformation

$$
F(z)=\sum_{x=0}^{\infty} \frac{f(x)}{z^{x}}
$$

is the powerful method to study discrete dynamic systems and generating functions

$$
F(z)=\sum_{x=0}^{\infty} f(x) z^{x}
$$

are used for studying problems in enumerative combinatorial analysis.

In the multi-dimensional case, the number of formulations of Cauchy problem (1)-(2) increases. We discuss some of them.

An analogue of the one-dimensional case (ii), when $X=\mathbb{Z}_{\geqslant}^{n}$ is the non-negative octant in $\mathbb{Z}^{n}$, $X_{0}=\mathbb{Z}_{\geqslant}^{n} \backslash X_{m}, 0 \in \Omega, m_{i}=\max \left\{\omega_{i}: \omega_{i} \in \Omega, i=1, \ldots, n\right\}, m=\left(m_{1}, \ldots, m_{n}\right)$ and $X_{m}=\{x \in$ $\left.\mathbb{Z}_{\geqslant}^{n}: x_{i} \geqslant m_{i}, i=1, \ldots, m\right\}$, is considered in [2], which is devoted to multi-dimensional difference equations with constant coefficients and their use in enumerative combinatorial analysis. Several equivalent conditions, providing solvability of problem (1)-(2), are given in Theorem 3 in [2]. Particularly, the convex hull conv $\{\Omega \backslash\{0\}\} \cap \mathbb{R}_{\geqslant}^{n}$ is not empty.

Various analogues of variant (i) of the Cauchy problem for the multi-dimensional case are constructed as follows. Let $A=\left\{\alpha^{1}, \ldots, \alpha^{N}\right\}$ be the set of vectors $\alpha^{j}=\left(\alpha_{1}^{j}, \ldots, \alpha_{n}^{j}\right) \in \mathbb{Z}^{n}$, $j=1, \ldots, N$, and $K$ is a lattice cone spanned by these vectors

$$
K=\left\{x \in \mathbb{Z}^{n}: x=\lambda_{1} \alpha^{1}+\cdots+\lambda_{N} \alpha^{N}, \lambda_{i} \in \mathbb{Z}_{\geqslant}, i=1, \ldots, N\right\}
$$

For points $u, v \in K$ a partial order relation $\underset{K}{\geqslant}$ is defined as follows: $u \geqslant v \Leftrightarrow u-v \in K$. We also denote $u \ngtr v \Leftrightarrow u-v \notin K$. We assume that the cone $K$ is pointed, which means it does not contain any line or, equivalently, lies in an open half-space of $\mathbb{R}^{n}$.

We consider a finite set of integer points $A \subset K$, in which there exists a point $m$ such that for all $\alpha^{j} \in A, j=1, \ldots, N$ the condition $\alpha^{j} \underset{K}{\leqslant} m$ holds. 
The solvability of the problem when the cone $K$ is simplicial (which means that every element in it admits a unique expansion in the generators) and the sets $X=K$ and $X_{0}=X \backslash(m+K)$, on which Cauchy problem (1)-(2) is solved, was studied in $[1,10-13,19]$. Additionally, in these papers, the solutions $f(x)$ to problem (1)-(2) are given in terms of the Cauchy data and fundamental solution to (1)-(2) (the Green function). These solutions play an important role in the study of asymptotics of solutions to the Cauchy problem, in particular, to study the stability of the problem and its connection with the properties of the characteristic set $\mathcal{V}_{P}:=\left\{z \in \mathbb{C}^{n}: P(z):=\sum_{\omega \in \Omega} c_{\omega} z^{\omega}=0\right\}$ of the equation (1), where $z^{\omega}=z_{1}^{\omega_{1}} \cdot \ldots \cdot z_{N}^{\omega_{N}}$.

A multidimensional analogue of option (ii) for the Cauchy problem(1)-(2) was not described in [2]. This is apparently due to the fact that in problems of enumerative combinatorial analysis the search for the generating function for the combinatorial object is considered as a full solution to the problem, rather than the study of its asymptotic behavior.

For $n>1$ we formulate the following variant of a Cauchy problem, which combines multidimensional analogs of (i) and (ii) for which the simplicity of the cone $K$ is not required. We denote $m=\alpha^{1}+\cdots+\alpha^{N}, c_{0}=1, \alpha^{0}=(0, \ldots, 0)$.

The Cauchy problem. Find a function $f: K \rightarrow \mathbb{C}$, satisfying the difference equation

$$
\sum_{j=0}^{N} c_{j} f\left(x-\alpha^{j}\right)=g(x), \quad x \underset{K}{\geqslant} m,
$$

and which coincides with the given function $\varphi(x)$ on the set $X_{0}=\{x \in K: x \underset{K}{\ngtr} m\}$ :

$$
f(x)=\varphi(x), \quad x \in X_{0} .
$$

The characteristic polynomial for (3) is a Laurent polynomial (since it may have terms of negative degree) $P(z)=\sum_{j=0}^{N} c_{j} z^{-\alpha^{j}}$.

Equation (3) with initial data (4) is used to describe a major class of problems in enumerative combinatorial analysis such as lattice path problems (the Dyck, Motzkin, Schröder and generalized lattice paths, see $[2,4,15])$.

The fact that the cone $K$ is pointed allows us to use the method of generating functions. This involves defining for any $\mu \in K$ the element in the ring $\mathbb{C}_{K}[[z]]$ of (formal) power series

$$
F_{\mu}(z)=\sum_{\substack{x \geqslant \mu \\ K}} f(x) z^{x}
$$

We also define $F(z)=F_{0}(z)$.

Using the method of generating functions, we will derive a formula which expresses the generating function $F(z)$ in terms of the characteristic polynomial for (3) and generating functions for the Cauchy data.

Theorem 1. The generating function $F(z)$ of a solution $f(x)$ to difference equation (3) with initial data (4) is representable as

$$
F(z)=\frac{1}{P\left(z^{-1}\right)}\left(\sum_{j=0}^{N} c_{j} z^{\alpha^{j}} \Phi_{m-\alpha^{j}}(z)+G_{m}(z)\right),
$$

where $P\left(z^{-1}\right)=P\left(z_{1}^{-1}, \ldots, z_{n}^{-1}\right), \Phi_{m-\alpha^{j}}(z)=F(z)-F_{m-\alpha^{j}}(z)$ and $G_{m}(z)=\sum_{x \geqslant m} g(x) z^{x}$. 
Proof. Multiplying the left-hand side of (3) by $z^{x}$ and summing over $x \geqslant m$ yields

$$
\begin{array}{r}
\sum_{x \geqslant m} \sum_{j=0}^{N} c_{j} f\left(x-\alpha^{j}\right) z^{x}=\sum_{j=0}^{N} c_{j} z^{\alpha^{j}} \sum_{x \geqslant m} f\left(x-\alpha^{j}\right) z^{x-\alpha^{j}}=\sum_{j=0}^{N} c_{j} z^{\alpha^{j}} \sum_{x+\alpha^{j} \geqslant m} f(x) z^{x}= \\
=\sum_{j=0}^{N} c_{j} z^{\alpha^{j}} F_{m-\alpha^{j}}(z)=\sum_{j=0}^{N} c_{j} z^{\alpha^{j}}\left(F(z)-\Phi_{m-\alpha^{j}}(z)\right) .
\end{array}
$$

Repeating the same with the right-hand side yields

$$
P\left(z^{-1}\right) \cdot F(z)=\sum_{j=0}^{N} c_{j} z^{\alpha^{j}} \Phi_{m-\alpha^{j}}(z)+G_{m}(z) .
$$

Thus we obtain (5), which proves the theorem.

Remark 1. Formulae (5) was derived in [14] for the Riordan arrays and in [11] for $K=\mathbb{Z}^{N}$ and $g(x)=0$.

A function $\mathcal{P}: \mathbb{Z}^{n} \rightarrow \mathbb{C}$ is called a fundamental solution to the Cauchy problem (3)-(4) if it satisfies to the difference equation

$$
\sum_{j=0}^{N} c_{j} \mathcal{P}\left(x-\alpha^{j}\right)=\delta_{0}(x), \quad x \in \mathbb{Z}^{n},
$$

where $\delta_{0}(x)$ is the Kronecker symbol:

$$
\delta_{0}(x)= \begin{cases}0 & \text { if } x \neq 0 \\ 1 & \text { if } x=0\end{cases}
$$

The support of the function $\mathcal{P}(x)$ is a set

$$
\operatorname{supp} \mathcal{P}(x)=\left\{x \in \mathbb{Z}^{n}: \mathcal{P}(x) \neq 0\right\} .
$$

Lemma. If $\mathcal{P}(x)$ is the fundamental solution to Cauchy problem (3)-(4) and $\operatorname{supp} \mathcal{P} \subset K$, where $K$ is a pointed cone, then

$$
P\left(z^{-I}\right) \cdot \sum_{x \in \mathbb{Z}^{n}} \mathcal{P}(x) z^{x}=1
$$

Proof. The product

$$
\sum_{j=0}^{N} c_{j} z^{\alpha^{j}} \cdot \sum_{x \in \mathbb{Z}^{n}} \mathcal{P}(x) z^{x}=\sum_{j=0}^{N} \sum_{x \in \mathbb{Z}^{n}} c_{j} \mathcal{P}(x) z^{x+\alpha^{j}}=\sum_{x \in \mathbb{Z}^{n}} \sum_{j=0}^{N} c_{j} \mathcal{P}\left(x-\alpha^{j}\right) z^{x}=\sum_{x \in \mathbb{Z}^{n}} \delta_{0}(x)=1,
$$

which proves the lemma.

The fundamental solution is

$$
\mathcal{P}(x)=\sum_{\substack{A \lambda=x \\ \lambda \in \mathbb{Z}_{\geqslant}^{N}}} \frac{\left(-c_{1}\right)^{\lambda_{1}} \cdot \ldots \cdot\left(-c_{N}\right)^{\lambda_{N}}\left(\lambda_{1}+\cdots+\lambda_{N}\right) !}{\lambda_{1} ! \ldots \lambda_{N} !}, \quad x \geqslant 0,
$$

and can be obtained by expanding $\frac{1}{P\left(z^{-1}\right)}$ into the Laurent series as follows: 


$$
\begin{aligned}
& \frac{1}{P\left(z^{-1}\right)}=\frac{1}{1-\sum_{j=1}^{N}\left(-c_{j}\right) z^{\alpha^{j}}}=\sum_{k=0}^{\infty}\left(\sum_{j=1}^{N}\left(-c_{j}\right) z^{\alpha^{j}}\right)^{k}= \\
& =\sum_{\lambda_{1}+\ldots+\lambda_{N} \geqslant 0} \frac{\left(-c_{1}\right)^{\lambda_{1}} \cdot \ldots \cdot\left(-c_{N}\right)^{\lambda_{N}}\left(\lambda_{1}+\cdots+\lambda_{N}\right) !}{\lambda_{1} ! \ldots \lambda_{N} !} z^{\lambda_{1} \alpha^{1}+\ldots+\lambda_{N} \alpha^{N}}= \\
& =\sum_{x \geqslant 0} \sum_{\substack{A \lambda=x \\
\lambda \in \mathbb{Z}_{\geqslant}^{N}}} \frac{\left(-c_{1}\right)^{\lambda_{1}} \cdot \ldots \cdot\left(-c_{N}\right)^{\lambda_{N}}\left(\lambda_{1}+\cdots+\lambda_{N}\right) !}{\lambda_{1} ! \ldots \lambda_{N} !} z^{x}=\sum_{\substack{x \geqslant 0 \\
K}} \mathcal{P}(x) z^{x} .
\end{aligned}
$$

The Laurent series $\sum_{\substack{x \geqslant 0 \\ K}} \mathcal{P}(x) z^{x}$ converges in a domain which can be described in term of an amoeba $\mathcal{A}_{P}$ of the Laurent polynomial $P(z)$. Namely, the logarithmic image of the domain is a complement component of the amoeba $\mathcal{A}_{P}$ corresponded with the point 0 of the Newtone polytope $\mathcal{N}_{P}$ (see [7]).

Function $P_{A}(x ; h)=\sum_{\substack{A \lambda=x \\ \lambda \in \mathbb{Z}_{\geqslant}^{N}}} h(\lambda)$ was considered in $[15]$ and called the vector partition function associated with $h(\lambda)$. Provided that $h(\lambda)=\frac{(-c)^{\lambda}|\lambda| !}{\lambda !}$, we get

$$
\mathcal{P}(x)=P_{A}(x ; h) .
$$

For $h(\lambda) \equiv 1$ the vector partition function $P_{A}(x ; h)=P_{A}(x)$ is a number of non-negative integer solutions to a linear Diophantine equation $A \lambda=x$ (see, for example, [17]):

$$
P_{A}(x)=\sum_{\substack{A \lambda=x \\ \lambda \in \mathbb{Z}^{N}}} 1, x \in \mathbb{Z}^{n} .
$$

For $h(\lambda)=e^{-\langle\lambda, y\rangle}$ properties of the function

$$
P_{A}(y ; h)=\sum_{\substack{A \lambda=x \\ \lambda \in \mathbb{Z}_{\geqslant}^{N}}} e^{-\langle\lambda, y\rangle}, y \in \mathbb{C}^{N},
$$

called the vector partition function associated with the set of vectors $A$, were investigated in [3]. In particular, they derive the residue formulas for its generating function and an analog of the Euler-Maclaurin formula, in which the vector partition functions are represented as the action of the Todd operator on the volume function of a polyhedron. Furthermore, a sum of $e^{-\langle\lambda, y\rangle}$ in integer cones was investigated in [16] in connection to generalization of the Riemann-Roch theorem. A structure theorem for the vector partition function was presented and polyhedral tools for the efficient computation of such functions was provided in [18].

For $\varphi(x) \equiv 1$ the function $P_{A}(\lambda ; \varphi)$ coincides with the classical vector partition function. For $\varphi(x)=e^{-\langle x, y\rangle}$ we obtain a vector partition function of the form (9). If we take $N=2, A=\left(\begin{array}{ll}1 & 1\end{array}\right)$ and $\varphi\left(x_{1}, x_{2}\right)=h\left(x_{1}\right)$, then $P_{A}(\lambda ; \varphi)=\sum_{\substack{x_{1}+x_{2}=\lambda \\ x_{1}, x_{2} \geqslant 0}} h\left(x_{1}\right)=\sum_{x_{1}=0}^{\lambda} h\left(x_{1}\right)$. Thus, the problem of finding the vector partition function $P_{A}(\lambda ; \varphi)$ is a generalization of the classical summation's problem of functions of a discrete argument.

The concept of the fundamental solution $\mathcal{P}(x)$ to (3)-(4) yields a formula expressing $f(x)$ in terms of Cauchy data $\varphi(x)$ and $g(x)$. 
Theorem 2. A solution to the difference equation (3) with initial data (4) is given as follows:

$$
\begin{gathered}
f(x)=\sum_{\substack{0 \leqslant y \leqslant x \\
K}} \mathcal{P}(x-y) \tau(y), \\
\text { where } \tau(y)= \begin{cases}\sum_{j=0}^{N} c_{j} \varphi\left(y-\alpha^{j}\right) & \text { if } y \underset{K}{\not} m, \\
g(y) & \text { if } y \underset{K}{\geqslant} m .\end{cases}
\end{gathered}
$$

Proof. Using expression (5) from Theorem 1 and expression (7) from Lemma yields

$$
F(z)=\sum_{\substack{x \geqslant 0 \\ K}} \mathcal{P}(x) z^{x}\left(\sum_{j=0}^{N} c_{j} z^{\alpha^{j}} \Phi_{m-\alpha^{j}}(z)+G_{m}(z)\right) .
$$

Since

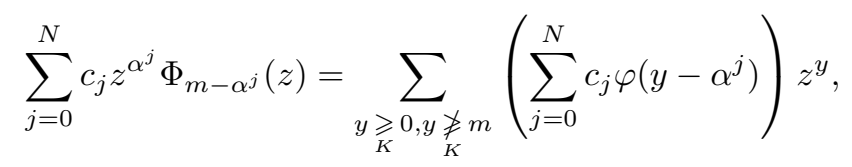

we get

$$
F(z)=\sum_{\substack{x \geqslant 0 \\ K}} \mathcal{P}(x) z^{x} \sum_{\substack{y \geqslant 0 \\ K}} \tau(y) z^{y}
$$

where $\tau(y)=\left\{\begin{array}{lc}\sum_{j=0}^{N} c_{j} \varphi\left(y-\alpha^{j}\right) & \text { if } y \ngtr m, \\ g(y) & \text { if } y \geqslant m .\end{array}\right.$

Finally, taking into account that $\mathcal{P}(x)=0$ for $\underset{K}{x} \underset{K}{\not}$ we get

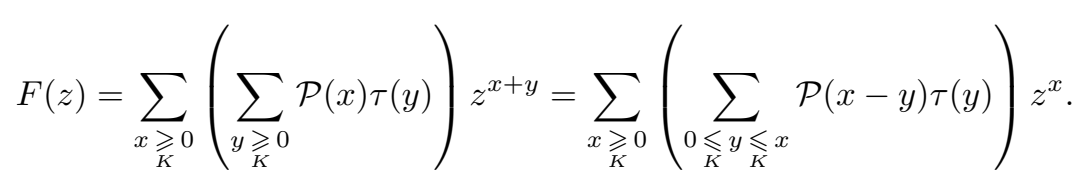

Equating the coefficients of $z^{x}$ we obtain

$$
f(x)=\sum_{\substack{0 \leqslant y \leqslant x \\ K}} \mathcal{P}(x-y) \tau(y),
$$

which proves the theorem.

\section{Applications to lattice path problems}

A lattice path is a finite sequence $p_{0}, p_{1}, \ldots, p_{L}$ of points in $\mathbb{Z}^{n}$ and its steps are the finite set of lattice vectors $p_{k}-p_{k-1} \in A=\left\{\alpha^{1}, \ldots, \alpha^{N}\right\}, k=1,2, \ldots, L$. The common class of lattice paths arises by imposing some conditions on the paths: points $p_{k}, k=0,1, \ldots, L$, are distinct (non intersecting paths). In the context of lattice path counting problems the function $f: \mathbb{Z}^{N} \rightarrow \mathbb{Z} \geqslant$ that counts the number $f(x)$ of paths in a specified class for which $p_{0}=0$ is computed (the 
condition $p_{0}=0$ does not result in a loss of generality). Examples of some well-known lattice paths: Dyck, Motzkin and Schröder paths (for more details see $[2,5,8,9]$ ).

It is well-known that the function $f(x)$ satisfies difference equation (3) with $c_{0}=1, c_{1}=$ $\ldots=c_{N}=-1$ and $g(x)=0$ (see [2]). Thus $P(\delta)=1-\delta^{-\alpha^{1}}-\cdots-\delta^{-\alpha^{N}}$.

Theorem 2 yields a simple formula for the number $f(x)$ of such paths (see also [15]). The following condition for an initial data function $\varphi(x)$ of Cauchy problem (3)-(4) for the lattice path problem holds:

$$
\varphi(x)= \begin{cases}0 & \text { if } x \neq 0, \\ 1 & \text { if } x=0, \\ (1-P(\delta)) \varphi(x) & \text { if } x \geqslant 0, x \neq 0 .\end{cases}
$$

Since $\tau(y)$ is equal to 1 only at the origin and vanishes at other points we get $f(x)=\mathcal{P}(x)$. Considering (8) we obtain

$$
f(x)=P_{A}(x ; h), \text { where } h(\lambda)=\frac{|\lambda| !}{\lambda !} .
$$

\section{Example A.}

We consider a set with three steps $A=\left\{\alpha^{1}=(1,0), \alpha^{2}=(0,1), \alpha^{3}=(1,1)\right\}$ and let $f\left(x_{1}, x_{2}\right)$ denote the number of paths from the origin to $\left(x_{1}, x_{2}\right) \in \mathbb{Z}^{2}$ using steps from the set $A$. The cone $K$ is spanned by the vectors from $A$ and $m=\alpha_{1}+\alpha_{2}$, since $\alpha_{3}=\alpha_{1}+\alpha_{2}$.

We consider the two dimensional difference equation

$$
f\left(x_{1}, x_{2}\right)-f\left(x_{1}-1, x_{2}\right)-f\left(x_{1}, x_{2}-1\right)-f\left(x_{1}-1, x_{2}-1\right)=0,
$$

and its characteristic polynomial $P\left(z_{1}, z_{2}\right)=1-z_{1}^{-1}-z_{2}^{-1}-z_{1}^{-1} z_{2}^{-1}$.

By Theorem 2 a solution to this difference equation is

$$
f\left(x_{1}, x_{2}\right)=\sum_{\substack{0 \leqslant y \leqslant x \\ K}} \mathcal{P}\left(x_{1}-y_{1}, x_{2}-y_{2}\right) \tau\left(y_{1}, y_{2}\right)
$$

where $\tau\left(y_{1}, y_{2}\right)=\varphi\left(y_{1}, y_{2}\right)-\varphi\left(y_{1}-1, y_{2}\right)-\varphi\left(y_{1}, y_{2}-1\right)-\varphi\left(y_{1}-1, y_{2}-1\right)$ if $\left(y_{1}, y_{2}\right) \ngtr(1,1)$ and $\tau\left(y_{1}, y_{2}\right)=0$ otherwise.

To find the fundamental solution $\mathcal{P}\left(x_{1}, x_{2}\right)$ we expand $P^{-1}\left(z_{1}^{-1}, z_{2}^{-1}\right)$ as follows

$$
\begin{aligned}
& \frac{1}{P\left(z_{1}^{-1}, z_{2}^{-1}\right)}=\frac{1}{1-\left(z_{1}+z_{2}+z_{1} z_{2}\right)}=\sum_{k=0}^{\infty}\left(z_{1}+z_{2}+z_{1} z_{2}\right)^{k}= \\
& \quad=\sum_{k_{1}, k_{2}, k_{3} \geqslant 0} \frac{\left(k_{1}+k_{2}+k_{3}\right) !}{k_{1} ! k_{2} ! k_{3} !} z_{1}^{k_{1}} z_{2}^{k_{2}}\left(z_{1} z_{2}\right)^{k_{3}}=\sum_{x_{1}, x_{2} \geqslant 0} \sum_{t=0}^{\min \left(x_{1}, x_{2}\right)} \frac{\left(x_{1}+x_{2}-t\right) !}{\left(x_{1}-t\right) !\left(x_{2}-t\right) ! t !} z_{1}^{x_{1}} z_{2}^{x_{2}} .
\end{aligned}
$$

Consequently, Lemma and the term of the fundamental solution gives

$$
\mathcal{P}\left(x_{1}, x_{2}\right)=\sum_{t=0}^{\min \left(x_{1}, x_{2}\right)} \frac{\left(x_{1}+x_{2}-t\right) !}{\left(x_{1}-t\right) !\left(x_{2}-t\right) ! t !}=\sum_{\substack{k_{1}+k_{3}=x_{1} \\ k_{2}+k_{3}=x_{2} \\ k_{1}, k_{2}, k_{3} \geqslant 0}} \frac{\left(k_{1}+k_{2}+k_{3}\right) !}{k_{1} ! k_{2} ! k_{3} !}=P_{A}(x ; \lambda) .
$$


Finally, we have the solution for difference equation (10) with initial data function $f\left(x_{1}, x_{2}\right)=$ $=\varphi\left(x_{1}, x_{2}\right),\left(x_{1}, x_{2}\right) \ngtr(1,1)$ as follows

$$
\begin{aligned}
f\left(x_{1}, x_{2}\right)=\mathcal{P}\left(x_{1}, x_{2}\right) \varphi(0,0)+\sum_{y_{1}=1}^{x_{1}} \mathcal{P}\left(x_{1}-y_{1}, x_{2}\right)\left(\varphi\left(y_{1}, 0\right)-\varphi\left(y_{1}-1,0\right)\right)+ & \\
& +\sum_{y_{2}=1}^{x_{2}} \mathcal{P}\left(x_{1}, x_{2}-y_{2}\right)\left(\varphi\left(0, y_{2}\right)-\varphi\left(0, y_{2}-1\right)\right) .
\end{aligned}
$$

In the case of lattice paths, $\varphi\left(y_{1}, 0\right)-\varphi\left(y_{1}-1,0\right)=0$ for $y_{1} \geqslant 1, \varphi\left(0, y_{2}\right)-\varphi\left(0, y_{2}-1\right)=0$ for $y_{2} \geqslant 1$, and $\varphi(0,0)=1$, we obtain

$$
f\left(x_{1}, x_{2}\right)=\mathcal{P}\left(x_{1}, x_{2}\right) .
$$

\section{Example $B$.}

Let $\alpha^{1}=(2,-1), \alpha^{2}=(-1,2)$ be a column vectors, we let $\mathrm{K}$ denote the cone $K$ spanned by the vectors $K=\left\langle\alpha^{1}, \alpha^{2}\right\rangle, m=\alpha^{1}+\alpha^{2}=(1,1)$.

We consider the two dimensional difference equation

$$
f\left(x_{1}, x_{2}\right)-f\left(x_{1}-2, x_{2}+1\right)-f\left(x_{1}+1, x_{2}-2\right)=0
$$

and its characteristic polynomial $P\left(z_{1}, z_{2}\right)=1-z_{1}^{-2} z_{2}-z_{1} z_{2}^{-2}$.

By Theorem 2 a solution to this difference equation is

$$
\begin{gathered}
f\left(x_{1}, x_{2}\right)=\sum_{\substack{\leqslant \\
K} \leqslant x} \mathcal{P}\left(x_{1}-y_{1}, x_{2}-y_{2}\right) \tau\left(y_{1}, y_{2}\right), \\
\text { where } \tau\left(y_{1}, y_{2}\right)=\left\{\begin{array}{l}
\varphi\left(y_{1}, y_{2}\right)-\varphi\left(y_{1}-2, y_{2}+1\right)-\varphi\left(y_{1}+1, y_{2}-2\right) \text { if }\left(y_{1}, y_{2}\right) \underset{K}{\ngtr}(1,1), \\
0 \text { if }\left(y_{1}, y_{2}\right) \geqslant(1,1) .
\end{array}\right.
\end{gathered}
$$

To find a fundamental solution $\mathcal{P}\left(x_{1}, x_{2}\right)$ we expand the characteristic polynomial $P\left(z_{1}^{-1}, z_{2}^{-1}\right)$ into a series:

$$
\begin{aligned}
\frac{1}{1-z_{1}^{2} z_{2}^{-1}-z_{1}^{-1} z_{2}^{2}}=\sum_{k=0}^{\infty}\left(z_{1}^{2} z_{2}^{-1}+z_{1}^{-1} z_{2}^{2}\right)^{k}=\sum_{k_{1}+k_{2} \geqslant 0} \frac{\left(k_{1}+k_{2}\right) !}{k_{1} ! k_{2} !}\left(z_{1}^{2} z_{2}^{-1}\right)^{k_{1}}\left(z_{1}^{-1} z_{2}^{2}\right)^{k_{2}}= \\
=\sum_{k_{1}+k_{2} \geqslant 0} \frac{\left(k_{1}+k_{2}\right) !}{k_{1} ! k_{2} !} z_{1}^{2 k_{1}-k_{2}} z_{2}^{-k_{1}+2 k_{2}}=\sum_{\left(x_{1}, x_{2}\right) \geqslant 0} \frac{\left(x_{1}+x_{2}\right) !}{\left(\frac{2 x_{1}+x_{2}}{3}\right) !\left(\frac{x_{1}+2 x_{2}}{3}\right) !} z_{1}^{x_{1}} z_{2}^{x_{2}} .
\end{aligned}
$$

Consequently,

$$
\mathcal{P}\left(x_{1}, x_{2}\right)=\frac{\left(x_{1}+x_{2}\right) !}{\left(\frac{2 x_{1}+x_{2}}{3}\right) !\left(\frac{x_{1}+2 x_{2}}{3}\right) !} .
$$

Finally, we have the solution for difference equation (11) with arbitrary initial data

$$
\begin{gathered}
f\left(x_{1}, x_{2}\right)=\varphi\left(x_{1}, x_{2}\right), \quad \underset{K}{\left(x_{1}, x_{2}\right) \underset{K}{\not}(1,1)} \\
f\left(x_{1}, x_{2}\right)=\mathcal{P}\left(x_{1}, x_{2}\right) \varphi(0,0)+\sum_{t=1}^{x_{1}} \mathcal{P}\left(x_{1}-2 t, x_{2}+t\right)(\varphi(2 t,-t)-\varphi(2 t-2,-t+1))+ \\
+\sum_{t=1}^{x_{2}} \mathcal{P}\left(x_{1}+t, x_{2}-2 t\right)(\varphi(-t, 2 t)-\varphi(-t+1,2 t-2)) . \\
-194-
\end{gathered}
$$


In the case of lattice paths, $\varphi(2 t,-t)-\varphi(2 t-2,-t+1)=0$ for $t \geqslant 1, \varphi(-t, 2 t)-\varphi(-t+1,2 t-2)=$ $=0$ for $t \geqslant 1$, and $\varphi(0,0)=1$, we obtain

$$
f\left(x_{1}, x_{2}\right)=\mathcal{P}\left(x_{1}, x_{2}\right) .
$$

This work of author was financed by the PhD SibFU grant for support of scientific research no. 14 .

\section{References}

[1] M.S.Apanovich, E.K.Leinartas, J. Sib. Fed. Univ. Math. Phys, 10(2017), no. 2, 199-205. DOI: $10.26516 / 1997-7670.2018 .26 .3$

[2] M.Bousquet-Mélou, M.Petkovšek, Discrete Mathematics, 225(2000), 51-75.

[3] M.Brion, M.Vergne, J. American Math. Soc., Vol. 10, no. 4 (1997), pp. 797-833. DOI: $10.1090 /$ S0894-0347-97-00242-7

[4] S.Chandragiri, J. Sib. Fed. Univ. Math. Phys, 12(2019), no. 5, 551-559.

DOI: 10.17516/1997-1397-2019-12-5-551-559

[5] P.Duchon, Discrete Math, 225(2000), 121-135.

[6] S.N.Elaydi, An Introduction to Difference Equations, 3rd edn. Undergraduate Texts in Mathematics, Springer, New York, 2005.

[7] M.Forsberg, M.Passare, A.Tsikh, Advances in Mathematics, 151(2000), no. 1, 45-70.

[8] I.M.Gessel, J. Combin. Theory. Ser. A, 28(1980), 321-337.

[9] J.Labelle, Y.N.Yeh, Generalized Dyck paths, Discrete Math, 82(1990), 1-6.

[10] E.K.Leinartas, Siberian Mathematical Journal, 48(2007), no. 2, 268-272.

DOI: $10.1007 / \mathrm{s} 11202-007-0026-0$

[11] E.K.Leinartas, A.P.Lyapin, J. Sib. Fed. Univ. Math. Phys, 2(2009), no. 4, 449-455 (in Russian).

[12] E.K.Leinartas, T.I.Nekrasova, Siberian Mathematical Journal, 57(2016), no. 2, 98-112. DOI: $10.17377 /$ smzh.2016.57.108

[13] E.K.Leinartas, M.S.Rogozina, Siberian Mathematical Journal, 56(2015), no. 1, 92-100. DOI: $10.1134 /$ S0037446615010097

[14] A.P.Lyapin, J. Sib. Fed. Univ. Math. Phys, 2(2009), no. 2, 210-220.

[15] A.P.Lyapin, S.Chandragiri, Journal of Difference Equations and Applications, 25(2019), no. $7,1052-1061$. DOI: $10.1080 / 10236198.2019 .1649396$

[16] A.V.Pukhlikov, A.G.Khovanskii, St. Petersburg Mathematical Journal, 4(1993), no. 4, 789-812.

[17] R.Stanley, Enumerative combinatorics, Cambridge Univ. Press, Cambridge, 1999. 
[18] B.Sturmfels, Journal of Combinatorial Theory. Series A, 72(1995), 302-309.

DOI: $10.1016 / 0097-3165(95) 90067-5$

[19] T.I.Yakovleva, Siberian Mathematical Journal, 58(2017), no. 2, 363-372.

DOI: $10.1134 /$ S0037446617020185

\title{
Задача Коши для многомерного разностного уравнения в конусах целочисленной решетки
}

\author{
Александр П. Ляпин \\ Сибирский федеральный университет \\ Красноярск, Российская Федерация \\ Лесосибирский педагогический институт - филиал СФУ \\ Лесосибирск, Российская Федерация \\ Шрилатха Чандрагири \\ Сибирский федеральный университет \\ Красноярск, Российская Федерация
}

\begin{abstract}
Аннотация. В работе рассмотрен вариант задачи Коши для многомерного разностного уравнения с постоянными коэффициентами, возникающий с задачей о числе путей на целочисленной решетке в перечислительном комбинаторном анализе. Получена формула, выражающая производящую функцию решения задачи Коши через производящие функции данных Коши, и найдено решение задачи Коши через ее фундаментальное решение и данные Коши.
\end{abstract}

Ключевые слова: разностное уравнение, фундаментальное решение, производящая функция, пути Дика. 